\title{
A single and double mode approach to chaotic vibrations of a cylindrical shell with large deflection
}

\author{
Liming Dai ${ }^{\mathrm{a}, *}$, Qiang $\operatorname{Han}^{\mathrm{b}}$ and Mingzhe Dong ${ }^{\mathrm{c}}$ \\ ${ }^{a}$ Industrial Systems Engineering, University of Regina, 3737 Wascana Parkway, Regina, Saskatchewan, Canada \\ S4SOA2 \\ ${ }^{\mathrm{b}}$ Department of Mechanics, College of Traffic and Communications, South China University of Technology, \\ Guangzhou, China 510640 \\ ${ }^{c}$ Petroleum Systems Engineering, University of Regina, 3737 Wascana Parkway, Regina, Saskatchewan, Canada \\ S4SOA2
}

Received 13 October 2003

Revised 8 December 2003

\begin{abstract}
The chaotic vibrations of a cylindrical shell of large deflection subjected to two-dimensional exertions are studied in the present research. The dynamic nonlinear governing equations of the cylindrical shell are derived on the basis of single and double mode models established. Two different types of nonlinear dynamic equations are obtained with varying dimensions and loading parameters. The criteria for chaos are determined via Melnikov function for the single mode model. The chaotic motion of the cylindrical shell is investigated and the comparison between the single and double mode models is carried out. Results of the research show that the single mode method usually used may lead to incorrect conclusions under certain conditions. Double mode or higher order mode methods should be used in these cases.
\end{abstract}

Keywords: Galerkin principle, melnikov function, chaos, nonlinear dynamics, cylindrical shell, single and double mode methods, numerical analysis

\section{Introduction}

In recent years, chaos in nonlinear dynamic systems has aroused more and more interest in the field of theoretical and experimental mechanics. Chaotic motion is regarded as a natural extension of the study object in nonlinear vibration. In solid mechanics, the chaotic behavior of buckled beams is studied by numerous researchers [1-4], and motion of the beams has been well understood. Among the recent research, the periodic and chaotic behavior of a viscoelastic nonlinear bar subjected to harmonic excitations was investigated by Suire et al. [5] on the based of a dynamics model established with implementation of Galerkin principle. The periodic and chaotic response of a slender beam with an attached mass under vertical base excitation was also reported [6]. However, a significantly less number of archival publications is available in investigating the chaotic properties of plates and shells. The forced response of a nearly square plate, the nonlinear dynamics of a shallow arch, and the chaotic motion of a circular plate and a cylindrical shell are a few typical studies in mechanical and structural systems found in the research [7-9]. Moreover, the single mode method is usually employed in the analysis of nonlinear dynamic systems. A typical

\footnotetext{
*Corresponding author. E-mail: dailimli@uregina.ca.
} 


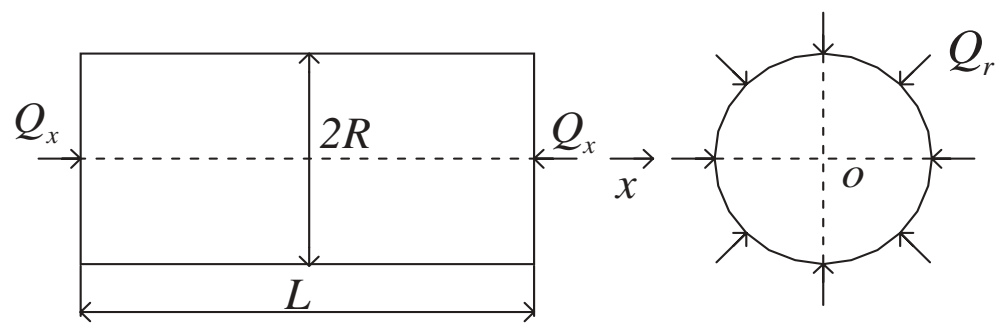

Fig. 1. A pinned shell.

example can be found from the article by Moon [2]. This article significantly contributes to the chaotic response of an elastic beam subjected to a periodic excitation with nonlinear boundary conditions and provided the criterion for chaos on the basis of a single mode model. In fact, most of the studies on nonlinear behavior of elastic elements utilize the single mode method. Based on the current literature, the differences between the single and double or higher mode methods have not been attended.

Cylindrical shells are widely used in civil, mechanical and petroleum engineering practices. However, a comprehensive understanding of the nonlinear behavior of the shells under dynamical loading is far from being reached. Among the available publications, for instance, a systematical and thorough study on elastic cylindrical shells of large deflection subjected to multi-axial exertions has not been found. The goal of the present research is to investigate the nonlinear behavior of an elastic cylindrical shell under excitations in longitudinal and radial directions. Large deflection of the shell is to be taken into consideration. Equations of motion for the cylindrical shell will be derived with both single and double modes. Nonlinear behavior of the shell, such as chaos, will be studied. The criteria for chaos of the cylindrical shell will be developed and the chaotic behavior of the transverse vibration of the shell will be investigated through a numerical analysis by the P-T method [10]. Results generated by single and double mode models will be compared and the differences of the two models will be identified and analyzed.

\section{Governing equations}

A pinned elastic cylindrical shell as shown in Fig. 1 will be studied. The cylindrical shell has diameter $2 R$, thickness $h$ and length $L$ and is subjected to uniformly distributed harmonic excitations, $Q_{x}$ and $Q_{r}$, in the longitudinal and radial directions respectively.

The excitations $Q_{x}$ and $Q_{r}$ are expressed in the following form:

$$
Q_{x}=q_{x}+q_{x 0} \cos \omega_{x} t, Q_{r}=q_{r}+q_{r 0} \cos \omega_{r} t
$$

The dynamic equation of the shell with large deflection can then be given in the following form with utilization of von Karman's theory for large deflection of shells [11].

$$
\begin{aligned}
& L(W, t)=D \nabla^{4} W-\frac{1}{R} \frac{\partial^{2} \varphi}{\partial x^{2}}-\frac{\partial^{2} \varphi}{\partial x^{2}} \cdot \frac{\partial^{2} W}{\partial y^{2}}+2 \cdot \frac{\partial^{2} \varphi}{\partial x \partial y} \cdot \frac{\partial^{2} W}{\partial x \partial y}-\frac{\partial^{2} \varphi}{\partial y^{2}}-\frac{\partial^{2} \varphi}{\partial y^{2}} \cdot \frac{\partial^{2} W}{\partial x^{2}}+\rho h \frac{\partial^{2} W}{\partial t^{2}} \\
& +c \frac{\partial^{2} W}{\partial t^{2}}+c \frac{\partial W}{\partial t}-Q_{r}=0 \\
& \nabla^{4} \varphi=E h\left[\left(\frac{\partial^{2} W}{\partial x \partial y}\right)^{2}-\frac{\partial^{2} W}{\partial x^{2}} \cdot \frac{\partial^{2} W}{\partial y^{2}}-\frac{1}{R} \frac{\partial^{2} W}{\partial x^{2}}\right]
\end{aligned}
$$

where

$$
D=\frac{E h^{3}}{12\left(1-\mu^{2}\right)},
$$




$$
\nabla^{4}=\frac{\partial^{4}}{\partial x^{4}}+2 \frac{\partial^{4}}{\partial x^{2} \partial y^{2}}+\frac{\partial^{4}}{\partial y^{4}}
$$

In Eqs (2a) and (2b), $W$ denotes the radial displacement, $\rho$ the density of the material, $c$ the damping coefficient, $\mu$ the Poisson ratio of the material, $E$ the elastic constant and $\varphi$ the stress function which gives the in-plane forces as follows.

$$
N_{x}=\frac{\partial^{2} \varphi}{\partial y^{2}}, N_{y}=\frac{\partial^{2} \varphi}{\partial x^{2}}, N_{x y}=-\frac{\partial^{2} \varphi}{\partial x \partial y}
$$

Following the Ritz method with two modes, one may have

$$
W(x, y, t)=W_{1}(t) \sin \frac{\pi x}{L} \sin \frac{\pi y}{R}+W_{2}(t) \sin \frac{2 \pi x}{L} \sin \frac{\pi y}{R}=W_{1} \sin \alpha x \sin \beta y+W_{2} \sin 2 \alpha x \sin \beta y
$$

where

$$
\alpha=\frac{\pi}{L}, \beta=\frac{\pi}{R}
$$

Trigonometric mode function is widely employed in describing the motion of a shell or plate [12]. Selection of the trigonometric mode function in the present research is based the consideration of the convenience of the functions in theoretical analysis for the response of the shell under the uniform loadings.

Substitution of Eq. (5) into Eq. (2b) gives

$$
\begin{aligned}
\nabla^{4} \varphi= & E h\left[\left(W_{1} \alpha \beta \cos \alpha x \cos \beta y+2 W_{2} \alpha \beta \cos 2 \alpha x \cos \beta y\right)^{2}-\left(-W_{1} \alpha^{2} \sin \alpha x \sin \beta y\right.\right. \\
& \left.-4 W_{2} \alpha^{2} \sin 2 \alpha x \sin \beta y\right) \cdot\left(-W_{1} \beta^{2} \sin \alpha x \sin \beta y-W_{2} \beta^{2} \sin 2 \alpha x \sin \beta y\right) \\
& \left.-\frac{1}{R}\left(-W_{1} \alpha^{2} \sin \alpha x \sin \beta-4 W_{2} \alpha^{2} \sin 2 \alpha x \sin \beta\right)\right] \\
= & E h\left[\frac{1}{2} \alpha^{2} \beta^{2} W_{1}^{2}(\cos 2 \alpha x+\cos 2 \beta y)+\frac{1}{4} \alpha^{2} \beta^{2} W_{1} W_{2}(-\cos \alpha x+9 \cos 3 \alpha x+9 \cos \alpha x \cos 2 \beta y\right. \\
& \left.-\cos 3 \alpha x \cos 2 \beta y)+2 \alpha^{2} \beta^{2} W_{2}^{2}(\cos 4 \alpha x+\cos 2 \beta y)+\frac{\alpha^{2}}{R}\left(W_{1} \sin \alpha x \sin \beta y+4 W_{2} \sin 2 \alpha x \sin \beta y\right)\right]
\end{aligned}
$$

The stress function $\varphi$ can be obtained as follows

$$
\begin{aligned}
\varphi= & E h\left[\frac{1}{2} \alpha^{2} \beta^{2} W_{1}^{2}\left(A_{1} \cos 2 \alpha x+A_{2} \cos 2 \beta y\right)+\frac{1}{4} \alpha^{2} \beta^{2} W_{1} W_{2}\left(A_{3} \cos \alpha x+A_{4} \cos 3 \alpha x\right.\right. \\
& +A_{5} \cos \alpha x \cos 2 \beta y+A_{6} \cos 3 \alpha x \cos 2 \beta y+2 \alpha^{2} \beta^{2} W_{2}^{2}\left(A_{7} \cos 4 \alpha x+A_{8} \cos 2 \beta y\right) \\
& \left.+\frac{\alpha^{2}}{R}\left(A_{9} W_{1} \sin \alpha x \sin \beta y+A_{10} W_{2} \sin 2 \alpha x \sin \beta y\right)\right]+\frac{y^{2}}{2} Q_{x}
\end{aligned}
$$

where

$$
\left\{\begin{array}{l}
A_{1}=\frac{1}{16 \alpha^{4}}, A_{2}=\frac{1}{16 \beta^{4}}, A_{3}=-\frac{1}{\alpha^{4}}, A_{4}=\frac{1}{9 \alpha^{4}}, A_{5}=\frac{9}{\left(\alpha^{2}+4 \beta^{2}\right)^{2}} \\
A_{6}=-\frac{1}{\left(9 \alpha^{2}+4 \beta^{2}\right)^{2}}, A_{7}=\frac{1}{256 \alpha^{4}}, A_{8}=\frac{1}{16 \beta^{4}}, A_{9}=\frac{1}{\left(\alpha^{2}+\beta^{2}\right)^{2}} \\
A_{10}=\frac{1}{\left(4 \alpha^{2}+\beta^{2}\right)^{2}}
\end{array}\right.
$$

Define the following shorthand notations:

$$
\left\{\begin{array}{l}
B_{1}=\frac{E h}{2} \alpha^{2} \beta^{2} A_{1}, B_{2}=\frac{E h}{2} \alpha^{2} \beta^{2} A_{2}, B_{3}=\frac{E h}{4} \alpha^{2} \beta^{2} A_{3}, B_{4}=\frac{E h}{4} \alpha^{2} \beta^{2} A_{4} \\
B_{5}=\frac{E h}{4} \alpha^{2} \beta^{2} A_{5}, B_{6}=\frac{E h}{4} \alpha^{2} \beta^{2} A_{6}, B_{7}=2 E h \alpha^{2} \beta^{2} A_{7}, B_{8}=2 E h \alpha^{2} \beta^{2} A_{8} \\
B_{9}=\frac{E h}{R} \alpha^{2} A_{9}, B_{10}=\frac{E h}{R} \alpha^{2} A_{10}
\end{array}\right.
$$

The stress function $\varepsilon$ can be rewritten as 


$$
\begin{aligned}
\varphi= & W_{1}^{2}\left(B_{1} \cos 2 \alpha x+B_{2} \cos 2 \beta y\right)+W_{1} W_{2}\left(B_{3} \cos \alpha x+B_{4} \cos 3 \alpha x+B_{5} \cos \alpha x \cos 2 \beta y\right. \\
& \left.+B_{6} \cos 3 \alpha x \cos 2 \beta y\right)+W_{2}^{2}\left(B_{7} \cos 4 \alpha x+B_{8} \cos 2 \beta y\right)+B_{9} W_{1} \sin \alpha x \sin \beta y \\
& +B_{10} W_{2} \sin 2 \alpha x \sin \beta y+\frac{y^{2}}{2} Q_{x}
\end{aligned}
$$

Substituting Eqs (5) and (11) into Eq. (2a), one may have

$$
\begin{aligned}
L(W)= & D\left[W_{1}\left(\alpha^{2}+\beta^{2}\right)^{2} \sin \alpha x \sin \beta y+W_{2}\left(4 \alpha^{2}+\beta^{2}\right)^{2} \sin 2 \alpha x \sin \beta y\right]-\left[W_{1}^{2}\left(-B_{1} \cdot 4 \alpha^{2} \cdot \cos 2 \alpha x\right)\right. \\
& +W_{1} W_{2}\left(-B_{3} \alpha^{2} \cos \alpha x-B_{4} \cdot 9 \alpha^{2} \cos 3 \alpha x-B_{5} \alpha^{2} \cos \alpha x \cos 2 \beta y-B_{6} \cdot 9 \alpha^{2} \cos 3 \alpha x \cos 2 \beta y\right) \\
& \left.+W_{2}^{2} \cdot\left(-B_{7} \cdot 16 \alpha^{2} \cos 4 \alpha x\right)-W_{1} B_{9} \cdot \alpha^{2} \sin \alpha x \sin \beta y-W_{2} \cdot B_{10} \cdot 4 \alpha^{2} \sin 2 \alpha x \sin \beta\right] \\
& \cdot\left[\frac{1}{R}+\left(-W_{1} \beta^{2} \sin \alpha x \cdot \sin \beta y-W_{1} \beta^{2} \sin 2 \alpha x \sin \beta y\right)\right]+2\left[W _ { 1 } W _ { 2 } \left(B_{5} \cdot 2 \alpha \beta \sin \alpha x \sin 2 \beta y\right.\right. \\
& \left.\left.+B_{6} \cdot 6 \alpha \beta \sin 3 \beta x \sin 2 \beta y\right)+W_{1} B_{9} \cdot \alpha \beta \cos \alpha x \cos \beta y+W_{2} \cdot B_{10} \cdot 2 \alpha \beta \cos 2 \alpha x \cos \beta y\right] \\
& \cdot\left[W_{1} \alpha \beta \cos \alpha x \cos \beta y+W_{2} 2 \alpha \beta \cos 2 \alpha x \cos \beta\right]-\left[W_{1}^{2}\left(-B_{2} \cdot 4 \beta^{2} \cos 2 \beta y\right)\right. \\
& +W_{1} W_{2}\left(-B_{5} \cdot 4 \beta^{2} \cos \alpha x \cos 2 \beta y-B_{6} \cdot 4 \beta^{2} \cos 3 \beta x \cos 2 \beta y\right)+W_{2}^{2}\left(-B_{8} \cdot 4 \beta^{2} \cos 2 \beta y\right) \\
& \left.-W_{1} B_{9} \sin \alpha x \sin \beta y-W_{2} B_{10} \beta^{2} \sin 2 \alpha x \sin \beta y+Q_{x}\right] \cdot\left[-W_{1} \alpha^{2} \sin \alpha x \sin \beta y\right. \\
& \left.-W_{2} \cdot 4 \alpha^{2} \sin 2 \alpha x \sin \beta y\right]+\rho h\left(\ddot{W}_{1} \sin \alpha x \sin \beta y+\ddot{W}_{2} \sin 2 \alpha x \sin \beta y\right) \\
& +c\left(\dot{W}_{1} \sin \alpha x \sin \beta y+\dot{W}_{2} \sin 2 \alpha x \sin \beta y\right)-Q_{r}=0
\end{aligned}
$$

Employing the Galerkin principle

$$
\left\{\begin{array}{l}
\iint L(W) \sin \alpha x \sin \beta y d x d y=0 \\
\iint L(W) \sin 2 \alpha x \sin \beta y d x d y=0,
\end{array}\right.
$$

the following nonlinear modal equations can be obtained.

$$
\left\{\begin{array}{l}
\dot{W}_{1}+m_{1} \dot{W}_{1}+m_{2} W_{1}^{3}+m_{3} W_{1}^{2}+m_{4} W_{1} W_{2}^{2}+m_{5} W_{1}+m_{6} W_{1}+m_{7} W_{2}^{2}+m_{8}=0 \\
\ddot{W}_{2}+n_{1} \dot{W}_{2}+n_{2} W_{2}^{3}+n_{3} W_{2} W_{1}^{2}+n_{4} W_{2} W_{1}+n_{5} W_{2}+n_{6} W_{2}=0
\end{array}\right.
$$

where

$$
\left\{\begin{array}{l}
m_{1}=\frac{c}{\rho h}, m_{2}=\frac{2 \alpha^{2} \beta^{2}}{\rho h}\left(B_{1}+B_{2}\right), m_{3}=-\frac{32 \alpha^{2}}{3 \pi^{2} \rho h R}\left(2 B_{1}+\beta^{2} B_{9} R\right) \\
m_{4}=\frac{\alpha^{2} \beta^{2}}{4 \rho h}\left(18 B_{4}-B_{6}-2 B_{3}+9 B_{5}+8 B_{8}\right), m_{5}=\frac{1}{\rho h R}\left(\alpha^{2} B_{9}+D\left(\alpha^{2}+\beta^{2}\right)^{2}\right) \\
m_{6}=\frac{\alpha^{2} Q_{x}}{\rho h}, m_{7}=-\frac{128}{15} \frac{\alpha^{2}}{\pi^{2} \rho h R}\left(2 B_{7}+3 \beta^{2} B_{10} R\right), m_{8}=-\frac{16 Q_{r}}{\pi^{2} \rho h} \\
n_{1}=\frac{c}{\rho h}, n_{2}=\frac{8 \alpha^{2} \beta^{2}}{\rho h}\left(B_{7}+B_{8}\right), n_{3}=\frac{\alpha^{2} \beta^{2}}{4 \rho h}\left(32 B_{2}+18 B_{4}-B_{6}+9 B_{5}-2 B_{3}\right) \\
n_{4}=-\frac{\alpha^{2}}{180 \pi^{2} \rho h R}\left(-3456 B_{6}+4608 \beta^{2} B_{9} R+640 B_{5}+10368 B_{4}+4608 \beta^{2} B_{10} R-1920 B_{3}\right) \\
n_{5}=\frac{1}{\rho h R}\left(D \beta^{4} R+16 D \alpha^{4} R+8 D \alpha^{2} \beta^{2} R+4 \alpha^{2} B_{10}\right), n_{6}=\frac{4 \alpha^{2} Q_{x}}{\rho h}
\end{array}\right.
$$

Let $W_{1}=c_{1} x_{1}, W_{2}=c_{2} x_{2}, t=c_{0} \tau, m_{5} c_{0}^{2}=1, m_{2} c_{0}^{2} c_{1}^{2}=1, n_{2} c_{0}^{2} c_{2}^{2}=1$, Eq. (14) can be written as

$$
\left\{\begin{array}{l}
\ddot{x}_{1}+\frac{m_{1}}{\sqrt{m_{5}}} \dot{x}_{1}+x_{1}^{3}+\frac{\sqrt{m_{3}}}{\sqrt{m_{2} m_{5}}} x_{1}^{2}+\frac{m_{4}}{n_{2}} x_{1} x_{2}^{2}+x_{1}+\frac{m_{6}}{m_{5}} x_{1}+\frac{m_{7}}{n_{2}} \sqrt{\frac{m_{2}}{m_{5}}} x_{2}^{2}+\frac{m_{8}}{m_{5}} \sqrt{\frac{m_{2}}{m_{5}}}=0 \\
\ddot{x}_{2}+\frac{n_{1}}{\sqrt{m_{5}}} \dot{x}_{2}+x_{2}^{3}+\frac{n_{3}}{m_{2}} x_{2} x_{1}^{2}+\frac{n_{4}}{\sqrt{m_{2} m_{5}}} x_{2} x_{1}+\frac{n_{5}}{m_{5}} x_{2}+\frac{n_{6}}{m_{5}} x_{2}=0
\end{array}\right.
$$




\section{Melnikov function for the single mode motion}

From Eq. (16), the following nonlinear dynamic equation can be constructed if only one mode is considered.

$$
\ddot{x}+\frac{m_{1}}{\sqrt{m_{5}}} \dot{x}+x^{3}+\frac{m_{3}}{\sqrt{m_{2} m_{5}}} x^{2}+x+\frac{m_{6}}{m_{5}} x+\frac{m_{8}}{m_{5}} \sqrt{\frac{m_{2}}{m_{5}}}=0
$$

Two cases are studied using the following common parameters.

$$
\left\{\begin{array}{l}
L=0.5 \mathrm{~m}, R=0.1 \mathrm{~m}, h=0.002 \mathrm{~m}, \alpha=\frac{\pi}{L} \mathrm{~m}^{-1}, \beta=\frac{\pi}{R} \mathrm{~m}^{-1} \\
E=69.7 \times 10^{9} \mathrm{~Pa}, \rho=2.78 \times 10^{3} \mathrm{~kg} / \mathrm{m}^{3}, \mu=0.3, c=1.67 \times 10^{7} \mathrm{~kg} \cdot\left(\mathrm{m}^{2} \cdot \mathrm{s}\right)^{-1} \\
q_{x}=0, q_{r 0}=0, q_{r}=g \times 1.56 \times 10^{10} \mathrm{~N} / \mathrm{m}^{2}
\end{array}\right.
$$

In the first case, $q_{x 0}=-10^{6} \mathrm{~N} / \mathrm{m}$, and Eq. (17) takes the following form

$$
\ddot{x}+0.5 x-3.8 x^{2}+x^{3}=g^{*} \cos \omega \tau-0.82 \dot{x}
$$

which in general can be expressed as

$$
\ddot{x}+x+\alpha x^{2}+\beta x^{3}=\varepsilon(g \cos \omega t-\mu \dot{x})
$$

where $\alpha, \beta$, and $\varepsilon$ are system parameters.

In the second case, $q_{x 0}=-4 \times 10^{6} \mathrm{~N} / \mathrm{m}$, and Eq. (17) reads

$$
\ddot{x}-x-3.8 x^{2}+x^{3}=g^{*} \cos \omega \tau-0.82 \dot{x}
$$

The corresponding general form of this system is

$$
\ddot{x}-x+\lambda_{2} x^{2}+\lambda_{3} x^{3}=\varepsilon(g \cos \omega t-\mu \dot{x})
$$

a) The Melnikov function of Eq. (20)

When $\varepsilon=0$, the corresponding unperturbed system is

$$
\left\{\begin{array}{l}
\dot{x}=y \\
\dot{y}=-x-\alpha x^{2}-\beta x^{3}
\end{array}\right.
$$

Its Hamilton function can be expressed as

$$
H(x, y)=\frac{1}{2} y^{2}+\frac{1}{2} x^{2}+\frac{\alpha}{3} x^{3}+\frac{\beta^{4}}{4} x^{4}
$$

The three fixed points are $(0,0)$ and $\left(\left(-\alpha \pm \sqrt{\alpha^{2}-4 \beta}\right) / 2 \beta, 0\right)$. For the homoclinic orbit of the system, $\left.x_{0}(t), y_{0}(t)\right)^{T}$, the Melnikov function [13] is defined as follows.

$$
M\left(t_{0}\right)=\int_{-\infty}^{+\infty} y_{0}(t)\left\{-\mu y_{0}(t)+g \cos \left[\bar{\omega}\left(t+t_{0}\right)\right]\right\} d t=-\mu A+g B\left(t_{0}\right)
$$

where

$$
\begin{aligned}
& A=\int_{-\infty}^{+\infty} y_{0}^{2}(t) d t \\
& B\left(t_{0}\right)=\int_{-\infty}^{+\infty} y_{0}(t) \cos \left[\bar{\omega}\left(t+t_{0}\right)\right] d t=\sqrt{B_{1}^{2}+B_{2}^{2}} \cos \left[\bar{\omega}\left(t+\tau_{0}\right)\right] \\
& B_{1}=\int_{-\infty}^{+\infty} y_{0}(t) \cos \bar{\omega} t d t, B_{2}=\int_{-\infty}^{+\infty} y_{0}(t) \sin \bar{\omega} t d t, \tau_{0}=\arctan \left(B_{2} / B_{1}\right) .
\end{aligned}
$$

One may therefore obtain

$$
M\left(t_{0}\right)=-\mu A+g \sqrt{B_{1}^{2}+B_{2}^{2}} \cos \left[\bar{\omega}\left(t_{0}+\tau_{0}\right)\right]
$$


The homoclinic orbit $\left.x_{0}(t), y_{0}(t)\right)^{T}$ can be determined by using the following equation

$$
H\left(\bar{x}_{0}, \bar{y}_{0}\right)=\frac{1}{2} y^{2}+\frac{1}{2} x^{2}+\frac{\alpha}{3} x^{3}+\frac{\beta}{4} x^{4}
$$

where $\left(\bar{x}_{0}, \bar{y}_{0}\right)^{T}$ is the saddle point, and we have

$$
d t / d x= \pm N^{-1}
$$

where

$$
N=\sqrt{2 H-x^{2}-\frac{2 \alpha}{3} x^{3}-\frac{\beta}{2} x^{4}}, H=H\left(\bar{x}_{0}, \bar{y}_{0}\right) .
$$

With these equations, $A, B_{1}$ and $B_{2}$ can be determined as

$$
\begin{aligned}
& |A|=\left|\int_{-\infty}^{+\infty} y_{0}^{2}(t) d t\right|=2\left|\int_{x(0)}^{x(+\infty)} N d x\right|,\left|B_{1}\right|=\left|\int_{-\infty}^{+\infty} y_{0}(t) \cos \bar{\omega} t d t\right|=0 \\
& \left|B_{2}\right|=\left|\int_{-\infty}^{+\infty} y_{0}(t) \sin \bar{\omega} t d t\right|=2\left|\int_{x(0)}^{x(+\infty)} \sin \left(\bar{\omega} \int_{x(0)}^{x} N^{-1} d s\right) d x\right|
\end{aligned}
$$

When the Melnikov function has simple zero points, the stable and unstable manifolds intersect. The Poincare map has a horseshoes; there therefore exists a strange constant set [14]. As such, it is possible for the dissipative system to enter chaos. According to the Melnikov method, the criterion for chaos can be determined as

$$
|g / \mu|>A / \sqrt{B_{1}^{2}+B_{2}^{2}}=\frac{\left|\int_{x(0)}^{x(+\infty)} N d x\right|}{\left|\int_{x(0)}^{x(+\infty)} \sin \left(\bar{\omega} \int_{x(0)}^{x} N^{-1} d s\right) d x\right|}
$$

b) The Melnikov function for Eq. (22)

Equation (22) can be rewritten in the following form

$$
\left\{\begin{array}{l}
\dot{x}=y \\
\dot{y}=x-\lambda_{2} x^{2}-\lambda_{3} x^{3}+\varepsilon(g \cos \omega t-\mu \dot{x})
\end{array}\right.
$$

Its unperturbed system is

$$
\ddot{x}-x+\lambda_{2} x^{2}+\lambda_{3} x^{3}=0 .
$$

This has the first integration

$$
\frac{1}{2} \dot{x}^{2}-\frac{1}{2} x^{2}+\frac{1}{3} \lambda_{2} x^{3}+\frac{1}{4} \lambda_{3} x^{4}=H
$$

With this expression, different values of $H$ indicate different curves in the corresponding phase portraits. The $H$ values are determined with the initial conditions. The three fixed points are $O, A$ and $B$, where $O$ is a hyperbolic-type fixed point and the others are stable fixed points, such that

$$
O(0,0), A\left(-\frac{-\lambda_{2}-\sqrt{\lambda_{2}^{2}+4 \lambda_{3}}}{2 \lambda_{3}}, 0\right), B\left(\frac{-\lambda_{2}+\sqrt{\lambda_{2}^{2}+4 \lambda_{3}}}{2 \lambda_{3}}, 0\right)
$$

Now let us find the homoclinic motion; with $H=0$. In this case

$$
\dot{x}= \pm \sqrt{x^{* 2}-\frac{2}{3} \lambda_{2} x^{* 3}-\frac{1}{2} \lambda_{3} x^{* 4}}
$$

The homoclinic orbit takes the following form.

$$
\left|\frac{\sqrt{1-\frac{2}{3} \lambda_{2} x^{*}-\frac{1}{2} \lambda_{3} x^{* 2}}-1}{x^{*}}-\frac{\lambda_{2}}{3}\right|=C_{2} e^{ \pm \sqrt{t}}
$$




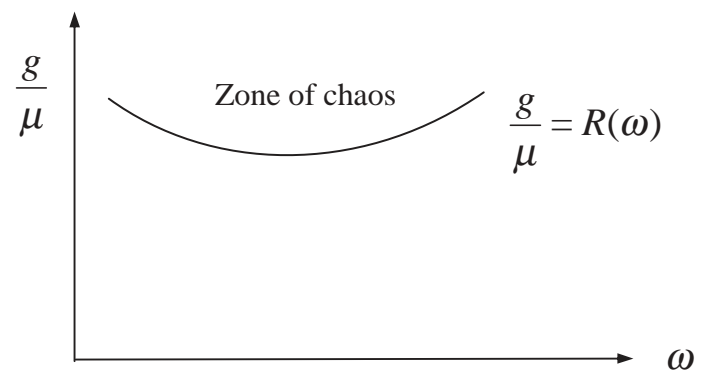

Fig. 2. Sketch of chaos criterion.

The corresponding Melnikov function is

$$
M(\tau)=\int_{-\infty}^{+\infty} \dot{x}^{*} \cdot \psi\left[x^{*}, \dot{x}^{*}, \omega(t+\tau)\right] d t
$$

where

$$
\Psi=\Psi(x, \dot{x}, \omega t)=g \cos \omega t-\mu \dot{x}
$$

therefore

$$
M(\tau)=-\mu \int_{-\infty}^{+\infty} \dot{x}^{* 2} d t+g \int_{-\infty}^{+\infty} \dot{x}^{*} \cos \omega(t+\tau) d t
$$

According to the residual law, the Melnikov function can be expressed as

$$
M(\tau)=-\frac{4 \mu}{3 \lambda_{3}}\left[1+\frac{\lambda_{2}^{2}}{3 \lambda_{3}}+\lambda_{2} \cdot \frac{2 \lambda_{2}^{2}+9 \lambda_{3}}{9 \lambda_{3} \sqrt{2 \lambda_{3}}} \arcsin \frac{\sqrt{2} \lambda_{2}}{\sqrt{2 \lambda_{2}^{2}+9 \lambda_{3}}}\right]+4 \pi g \omega \cdot \frac{\sin \omega(\xi+\tau)\left(\operatorname{ch} \omega \eta_{1}-\operatorname{ch} \omega \eta_{2}\right)}{\sqrt{2 \lambda_{3}}(\operatorname{ch} 2 \pi \omega-1)}
$$

where

$$
\left\{\begin{array}{l}
\xi=\ln \left[\frac{1}{3 C_{2}} \sqrt{\frac{2 \lambda_{2}^{2}+9 \lambda_{3}}{2}}\right] \\
\eta_{1}=\pi+\arctan \left(\frac{3}{\lambda_{2}} \sqrt{\frac{\lambda_{3}}{2}}\right) \\
\eta_{2}=\pi-\arctan \left(\frac{3}{\lambda_{2}} \sqrt{\frac{\lambda_{3}}{2}}\right)
\end{array}\right.
$$

where $C_{2}$ is a constant and can be determined by the initial conditions. When the Melnikov function has simple zero points, the nonlinear system may lead to a Smale horseshoe type of chaos. That implies that the criterion of chaos in this case should take the following form:

$$
\frac{g}{\mu} \geqslant \frac{2(\operatorname{ch} 2 \pi \omega-1)}{3 \sqrt{2} \omega \sqrt{\lambda_{3}}\left(\operatorname{ch} \omega \eta_{1}-c h \omega \eta_{2}\right)}\left[1+\frac{\lambda_{2}^{2}}{3 \lambda_{3}}+\lambda_{2} \cdot \frac{2 \lambda_{2}^{2}+9 \lambda_{3}}{9 \lambda_{3} \sqrt{2 \lambda_{3}}} \arcsin \frac{\sqrt{2} \lambda_{2}}{\sqrt{2 \lambda_{2}^{2}+9 \lambda_{3}}}\right]=R(\omega),
$$

This criterion is graphically demonstrated in Fig. 2.

As illustrated in Fig. 2 that chaos occurs when the value of $g / \mu$ is greater than $R(\omega)$ expressed in Eq. (46).

\section{Numerical simulations}

According to the nonlinear dynamic Eqs (16) and (17), the following numerical computations are carried out by the P-T method [10]. The parameters used are those as indicated in Eq. (18). The initial conditions of the numerical simulations for the single mode model are $x=0.0$, and $\dot{x}=0.0$ as $t=0$ For the double mode model, the initial conditions are $x_{1}=0.0, \dot{x}_{1}=0.0, x_{2}=0.00001, \dot{x}_{2}=0.0$ as $t=0$ 


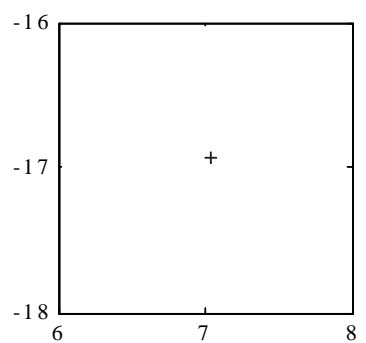

Poincare map

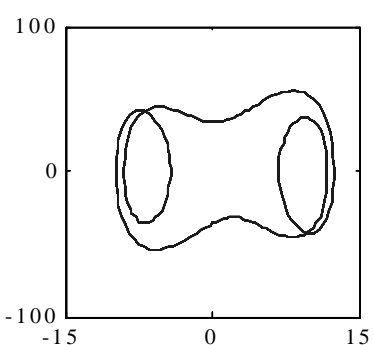

phase portrait

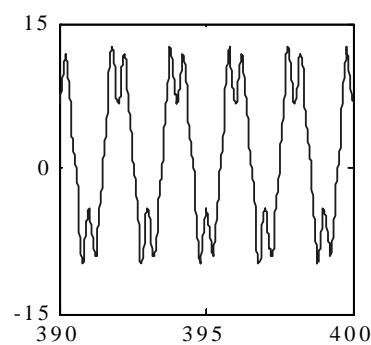

time history

(a) single mode model $x$

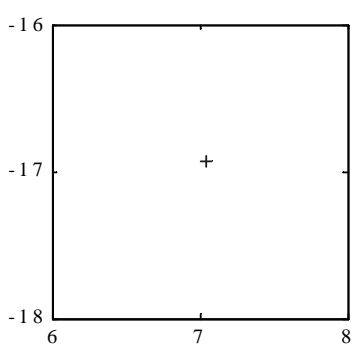

Poincare map

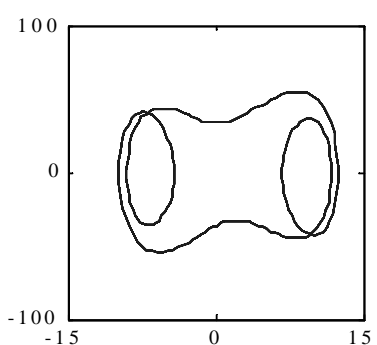

phase portrait

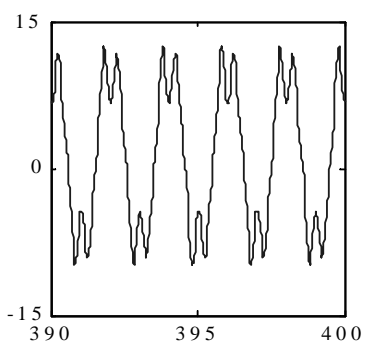

time history

(b) double mode model $x_{1}$

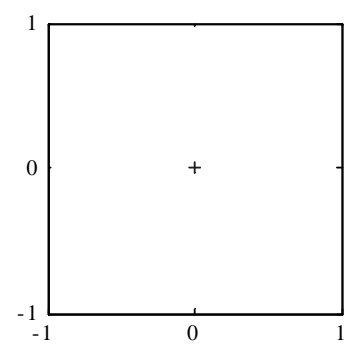

Poincare map

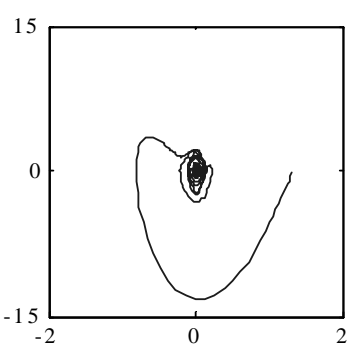

phase portrait

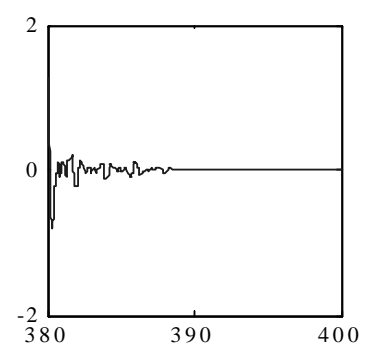

time history

(The vertical axis is amplified by $10^{65}$ times)

(c) double mode model $x_{2}$

Fig. 3. Comparison between single and double mode models $(\omega=\pi, g=610)$.

The displacement modes used in this paper based on the single and double mode models can be expressed as follows.

$$
\begin{aligned}
& w(x, y, t)=x(t) \sin \frac{\pi x}{L} \sin \frac{\pi y}{R}, \text { for the single mode model } \\
& W^{*}(x, y, t)=x_{1}(t) \sin \frac{\pi x}{L} \sin \frac{\pi y}{R}+x_{2}(t) \sin \frac{2 \pi x}{L} \sin \frac{\pi y}{R}, \text { for the double mode model }
\end{aligned}
$$




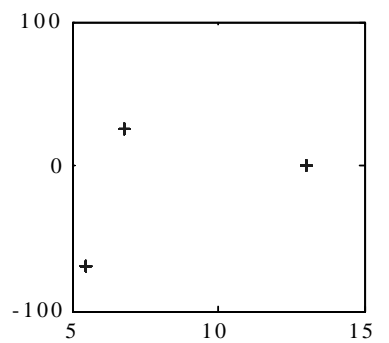

Poincare map

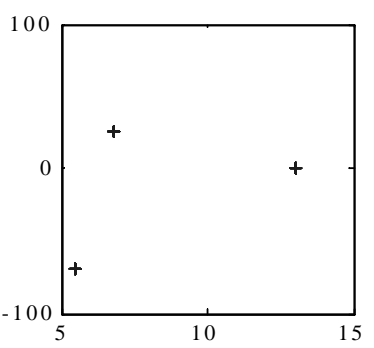

Poincare map

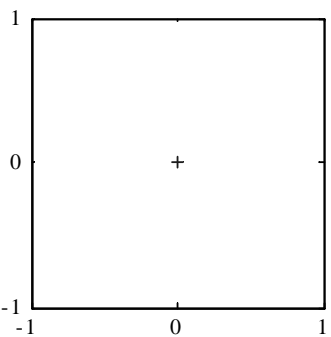

Poincare map

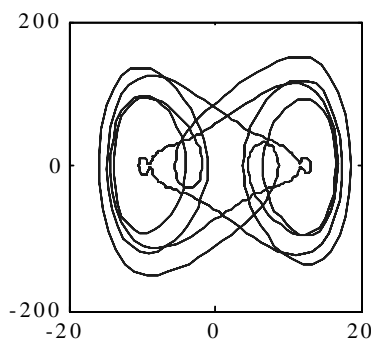

phase portrait

(a) single mode model $x$

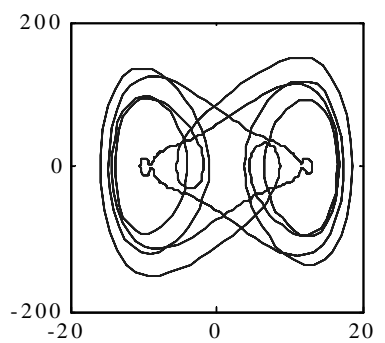

phase portrait

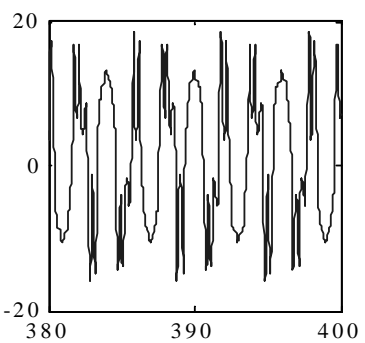

time history

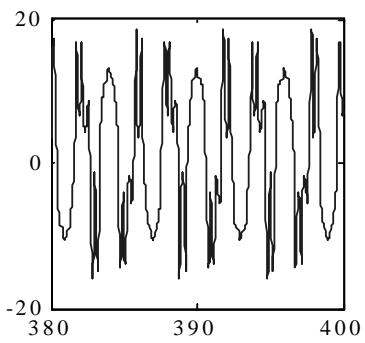

time history

(b) double mode model $x_{1}$

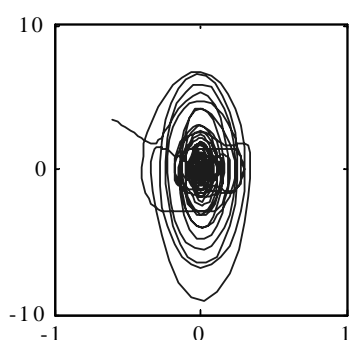

phase portrait

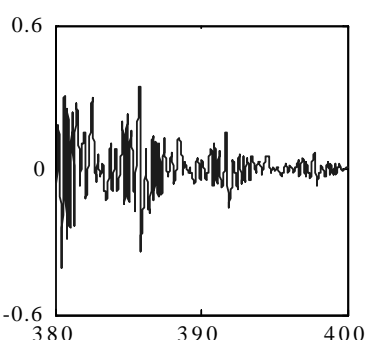

time history

(The vertical axis is amplified by $10^{21}$ times)

(c) double mode model $x_{2}$

Fig. 4. Comparison between single and double mode models $(\omega=\pi, g=1400)$.

If the single mode model is tenable, the following expression should be true.

$$
W(x, y, t) \approx W^{*}(x, y, t), x(t) \approx x_{1}(t),\left|\frac{x_{2}(t)-x_{1}(t)}{x_{1}(t)}\right|<<1
$$

As can be seen from Fig. 3 to Fig. 5, the results obtained from the single and double mode models are completely identical when, $\omega=\pi, g=610, \omega=\pi, g=1400$ and $\omega=\pi, g=1400$ if the other parameters are kept as the same. This is to say; in these cases the single mode model analysis is sufficient and correct. 


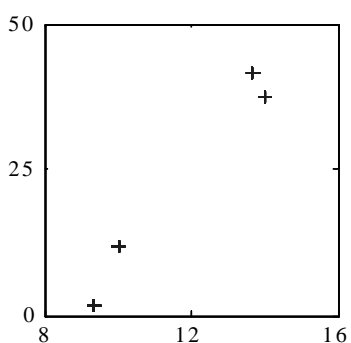

Poincare map

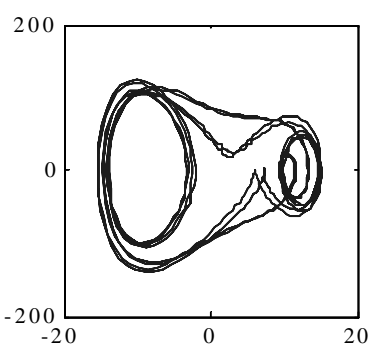

phase portrait

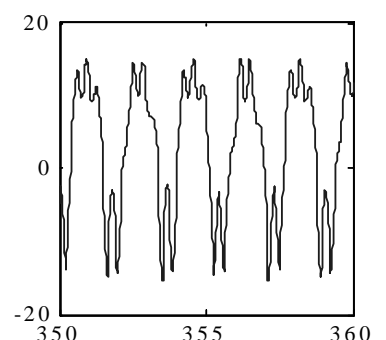

time history

(a) single mode model $x$

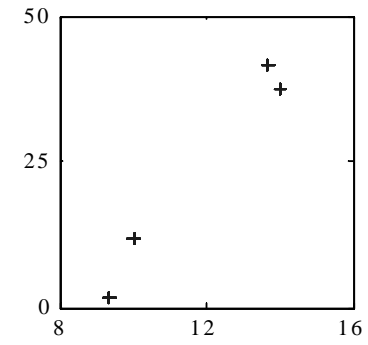

Poincare map

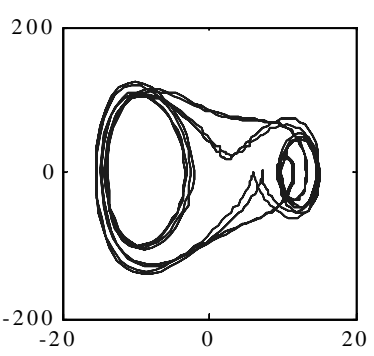

phase portrait

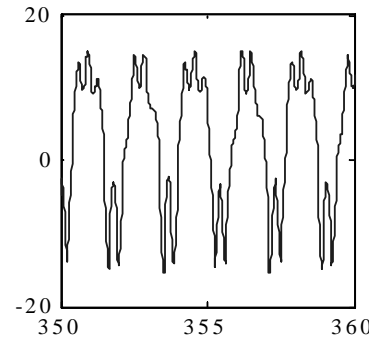

time history

(b) double mode model $x_{1}$

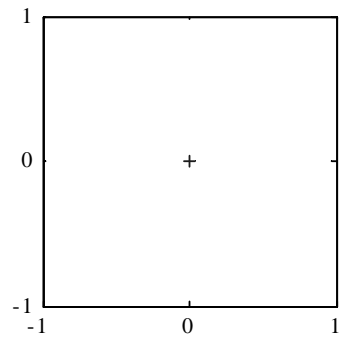

Poincare map

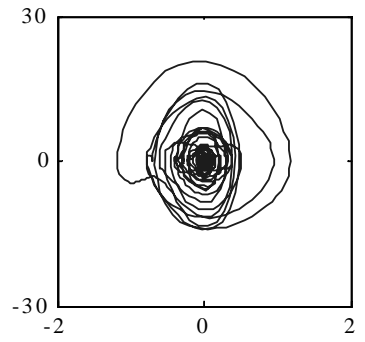

phase portrait

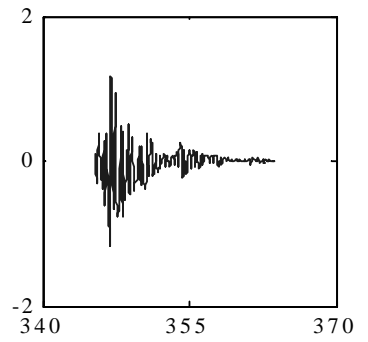

time history

(The vertical axis is amplified by $10^{32}$ times)

(c) double mode model $x_{2}$

Fig. 5. Comparison between single and double mode models $(\omega=1.1 \pi, g=1400)$.

As illustrated in Fig. 6, 7 and 8, the single and double mode models generate completely different results. Chaotic behavior of the motion is identified by the results of the double mode model for the cases in which $\left|\frac{x_{2}(t)-x_{1}(t)}{x_{1}(t)}\right|<<1$ is not satisfied; whereas the corresponding results created by the single mode model indicate periodic or quasiperiodic behavior as shown in the figures. In other words, the single mode method, which is widely used in the dynamic analysis, may lead to incorrect conclusions in these cases. It is therefore clear that the single mode method has limits in analyzing the elastic structure's nonlinear response. For the cases as indicated above, double mode or higher order mode method should be used for reliable results. 


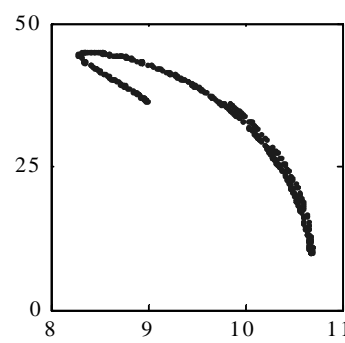

Poincare map

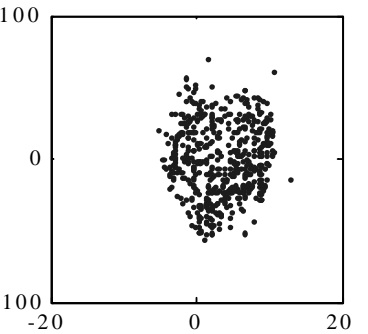

Poincare map

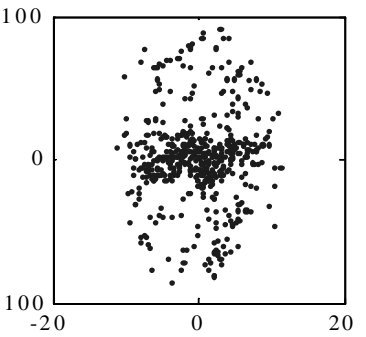

Poincare map

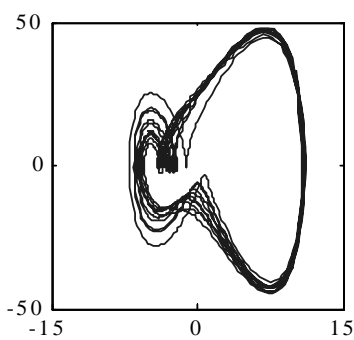

phase portrait

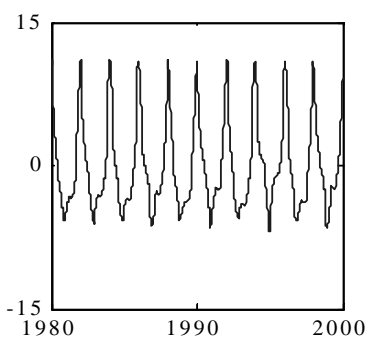

time history

(a) single mode $x$

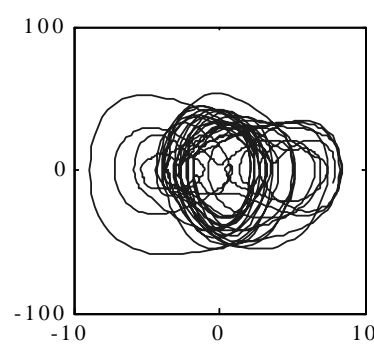

phase portrait

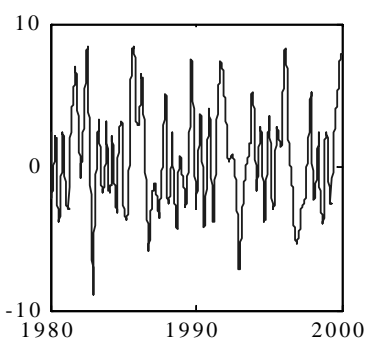

time history

(b) double mode $x_{1}$

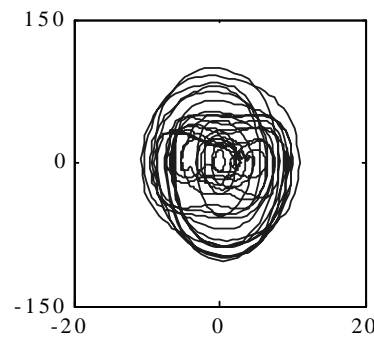

phase portrait

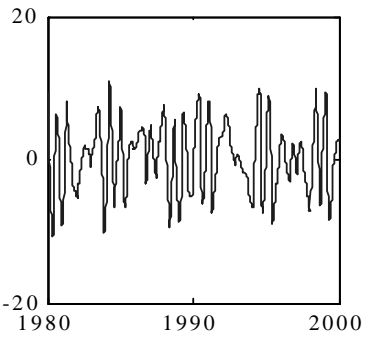

time history

(c) double mode $x_{2}$

Fig. 6. Comparison between single and double mode models $(\omega=\pi, g=200)$.

\section{Concluding remarks}

The characteristics of the nonlinear transverse vibration of an elastic cylindrical shell with large deflection and under uniform harmonic excitations are investigated in the present research based on the single and double mode models. In the current literature, systematical studies on such a shell exerted by multiple loadings are not found. Dynamic nonlinear modal equations for the motion of the cylindrical shell are derived on the basis of the models. Two types of nonlinear dynamic equations are obtained with a variety of system and loading parameters. Chaotic behavior of the cylindrical shell is evident as found in the present research. The criteria of chaos are determined for the motion of the cylindrical shell with the Melnikov function for the single mode model. Differences between the single and double mode models are apparent and a comparison between the results generated by the single and double mode models is carried out using numerical computations via the P-T method. 


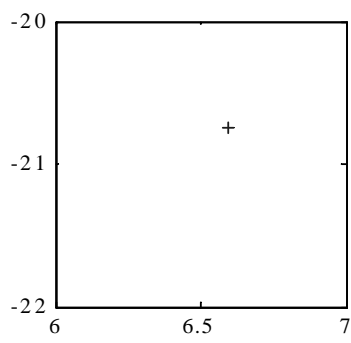

Poincare map

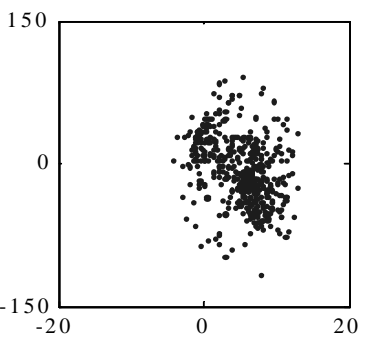

Poincare map

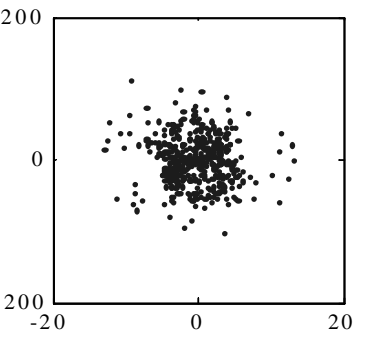

Poincare map

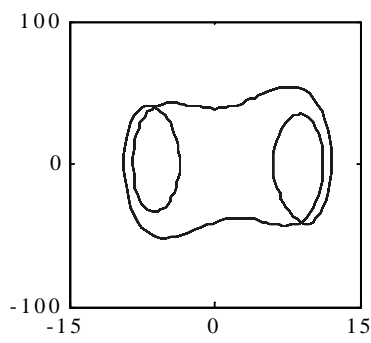

phase portrait

(a) single mode $x$

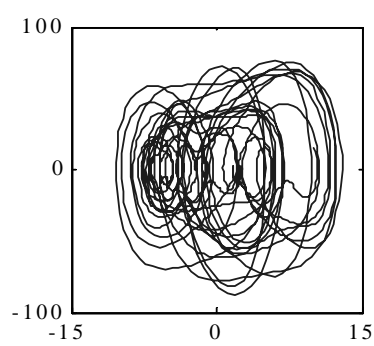

phase portrait

(b) double mode $x_{1}$

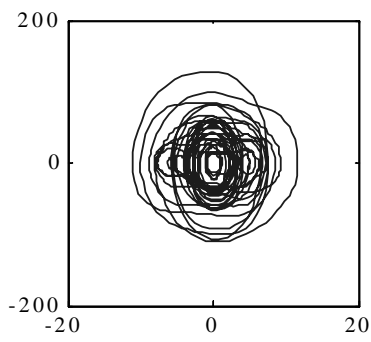

phase portrait

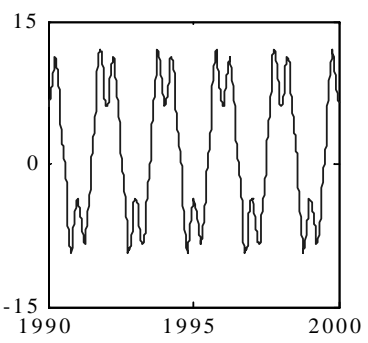

time history

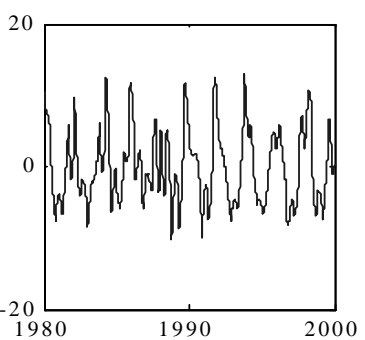

time history

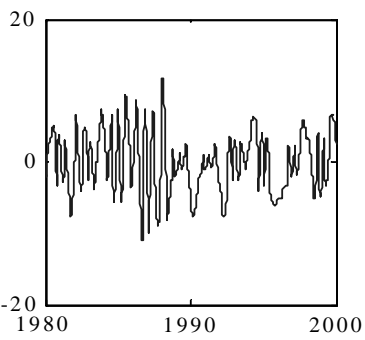

time history

(c) double mode $x_{2}$

Fig. 7. Comparison between single and double mode models $(\omega=\pi, g=500)$.

Based on the theoretical and numerical analyses of the present research, it can also be stated that $W_{2}(\xi, t)=$ $T_{1}(t) W_{1}^{*}(\xi)+T_{2}(t) W_{2}^{*}(\xi)$ of the double mode model is close to $W_{1}(\xi, t)=T(t) W_{1}^{*}(\xi)$ of the single mode model, provided that $\left|\frac{x_{2}(t)-x_{1}(t)}{x_{1}(t)}\right|<<1$ is complied. However, $w_{2}(\xi, t)$ is a better approximation solution over $W_{1}(\xi, t)$, when the conditions of $\left|\frac{T(t)}{T_{1}(t)}\right| \approx 1$ and $\left|\frac{T_{1}(t) W_{2}^{*}(\xi)-T_{1}(t) W_{1}^{*}(\xi)}{T_{1}(t) W_{1}^{*}(\xi)}\right|<<1$ are satisfied. In this case, if $T(t)$ is chaotic, $T_{1}(t)$ is also chaotic correspondingly, and vice versa. When the condition $\left|\frac{T_{2}(t) W_{2}^{*}(\xi)-T_{1}(t) W_{1}^{*}(\xi)}{T_{1}(t) W_{1}^{*}(\xi)}\right|<<1$ is not maintained, the single mode method, which is conventionally used in the literature for analyzing the nonlinear behavior of an elastic system, may lead to incorrect conclusions and is therefore no longer reliable. The double mode or higher order mode models should then be employed in these cases. 


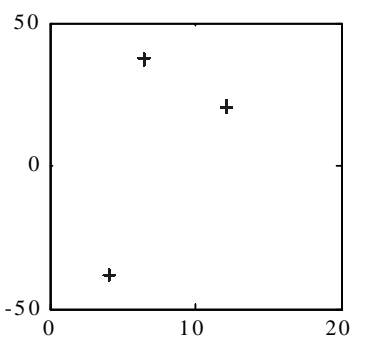

Poincare map

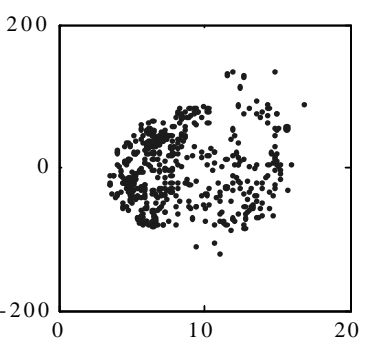

Poincare map

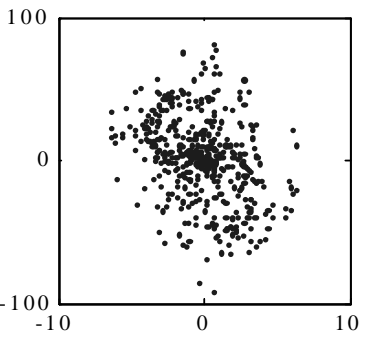

Poincare map

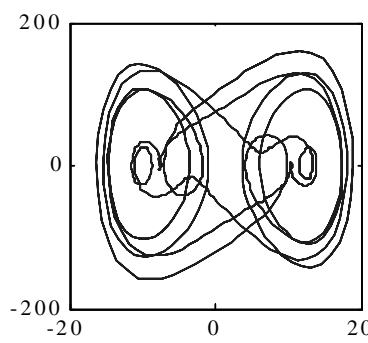

phase portrait

(a) single mode $x$

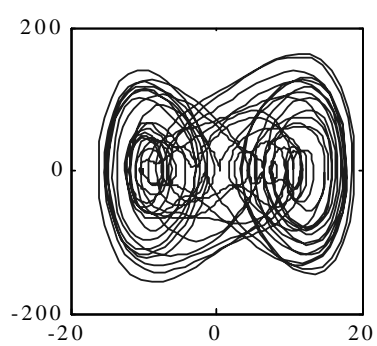

phase portrait

(b) double mode $\mathrm{x}_{1}$

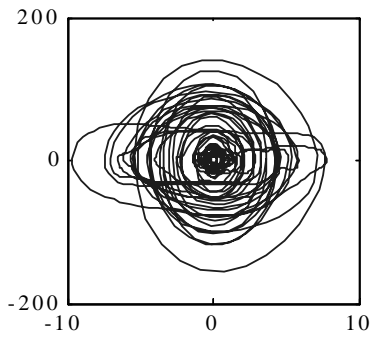

phase portrait

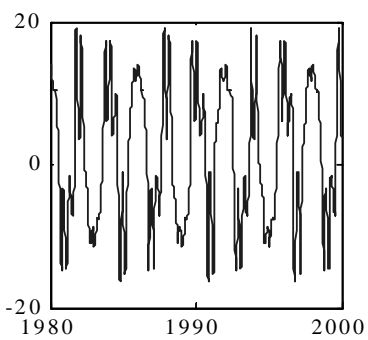

time history

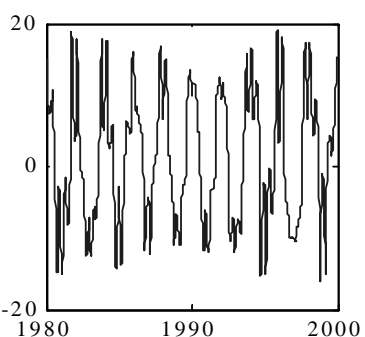

time history

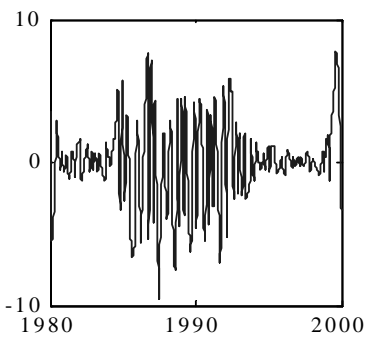

time history

(c) double mode $\mathrm{x}_{2}$

Fig. 8. Comparison between single and double mode models $(\omega=\pi, g=1500)$.

\section{Acknowledgement}

This research is supported by NSERC, CFI and NNSFC.

\section{References}

[1] P. Holms and J. Marsden, A Partial Differential Equation with Infinitely Many Periodic Orbits: Chaotic Oscillation of a Forced Beam, Arch. Rat. Mech. and Analysis 2 (1981), 135-165.

[2] F.C. Moon and S.W. Shaw, Chaotic Vibration of A Beam with Nonlinear Boundary Conditions, Non-linear Mech 18 (1983), $230-240$.

[3] P.D. Baran, Mathematical Models Used in Studying the Chaotic Vibration of Buckled Beam, Mechanics Research Communications 21 (1994), 189-196. 
[4] V. Keragiozov and D. Keoagiozova, Chaotic Phenomena in the Dynamic Buckling of an Elastic-Plastic Column Under an Impact, Nonlinear Dynamics 13 (1995), 1-16.

[5] G. Suire and G. Cederbaum, Periodic and Chaotic Behavior of Viscoelastic Nonlinear (Elastica) Bars under Harmonic Excitations 37 (1995), 753-772.

[6] S. Dwivedy and R. Kar, Dynamics of a Slender Beam with an Attached Mass under Combination Parametric and Internal Resonances, Part II: Periodic and Chaotic Responses, J. Sound Vib. 222 (1999), 281-305.

[7] X.L. Yang and P.R. Sethna, Nonlinear Forced Vibrations of a Nearly Square Plate-Antisymmetric Case, J. Sound Vib. 155 (1992), $413-441$.

[8] W. Tien, N. Namachchivaya and N. Malhotra, Non-Linear Dynamics of a Shallow Arch Under Periodic Excitation-II.1:1 Internal Resonance, Int. J. Non-Linear Mech. 29 (1994), 367-385.

[9] Q. Han, H.Y. Hu and G.T. Yang, A Study of Chaotic Motion in Elastic Cylindrical Shells, Eur. J. Mech. A/Solids 18 (1999), 351-360.

[10] L. Dai and M.C. Singh, A New Approach to Approximate and Numerical Solutions of Oscillatory Problems, J. Sound Vib. 263 (2003), $535-548$.

[11] T. von Karman, Festigkeits Probleme in Machinenbau, Encl. Der math. Wiss. 4 (1910), 348-351.

[12] A.C. Ugural, Stress in Plates and Shells, McGraw-Hill, Boston, 1999.

[13] S. Wiggins, Chaotic Transport in Dynamical Systems, Springer-Verlag, New York, 1992.

[14] R. Temam, Infinite-Dimensional Dynamical Systems in Mechanics and Physics, Springer, New York, 1997. 

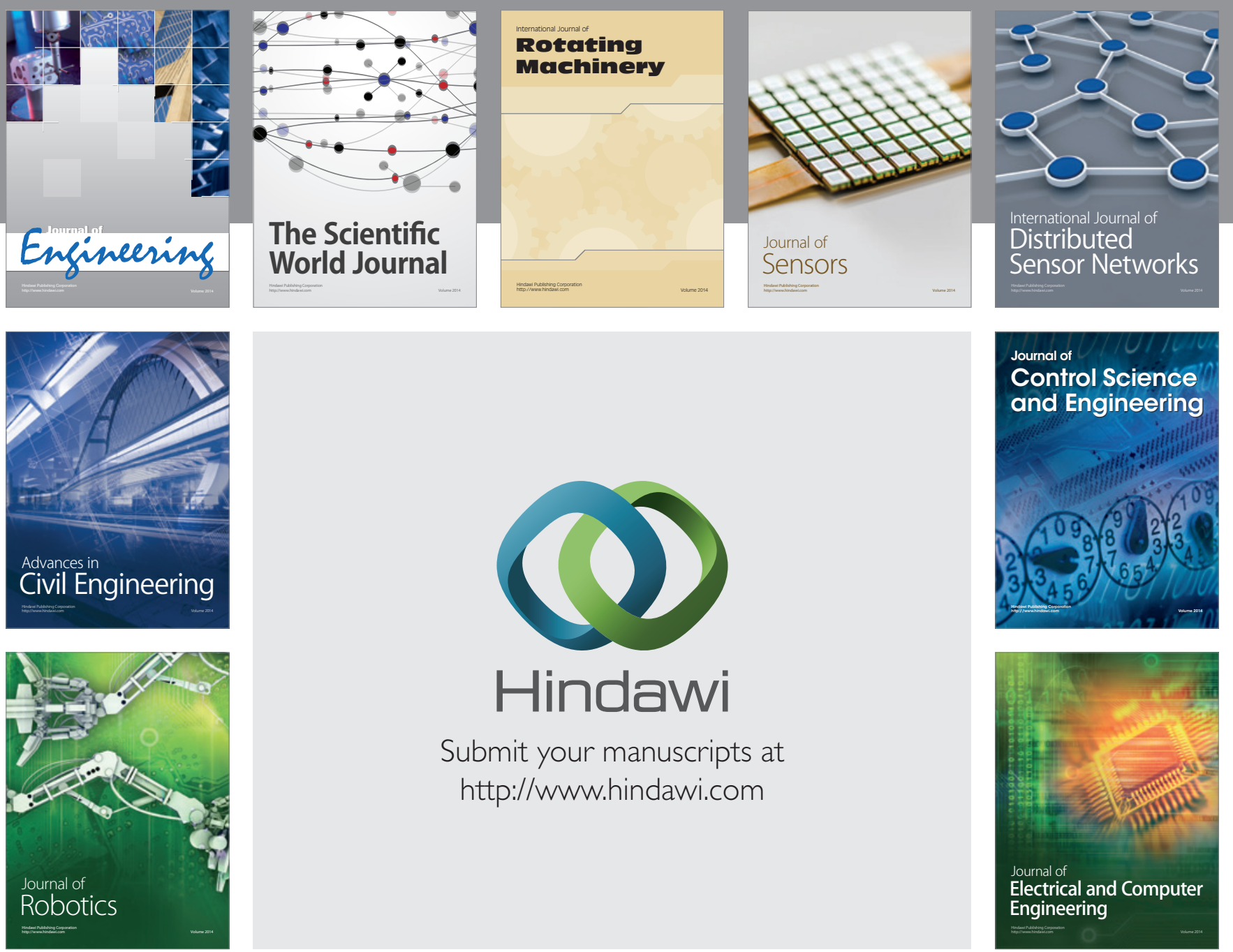

Submit your manuscripts at

http://www.hindawi.com
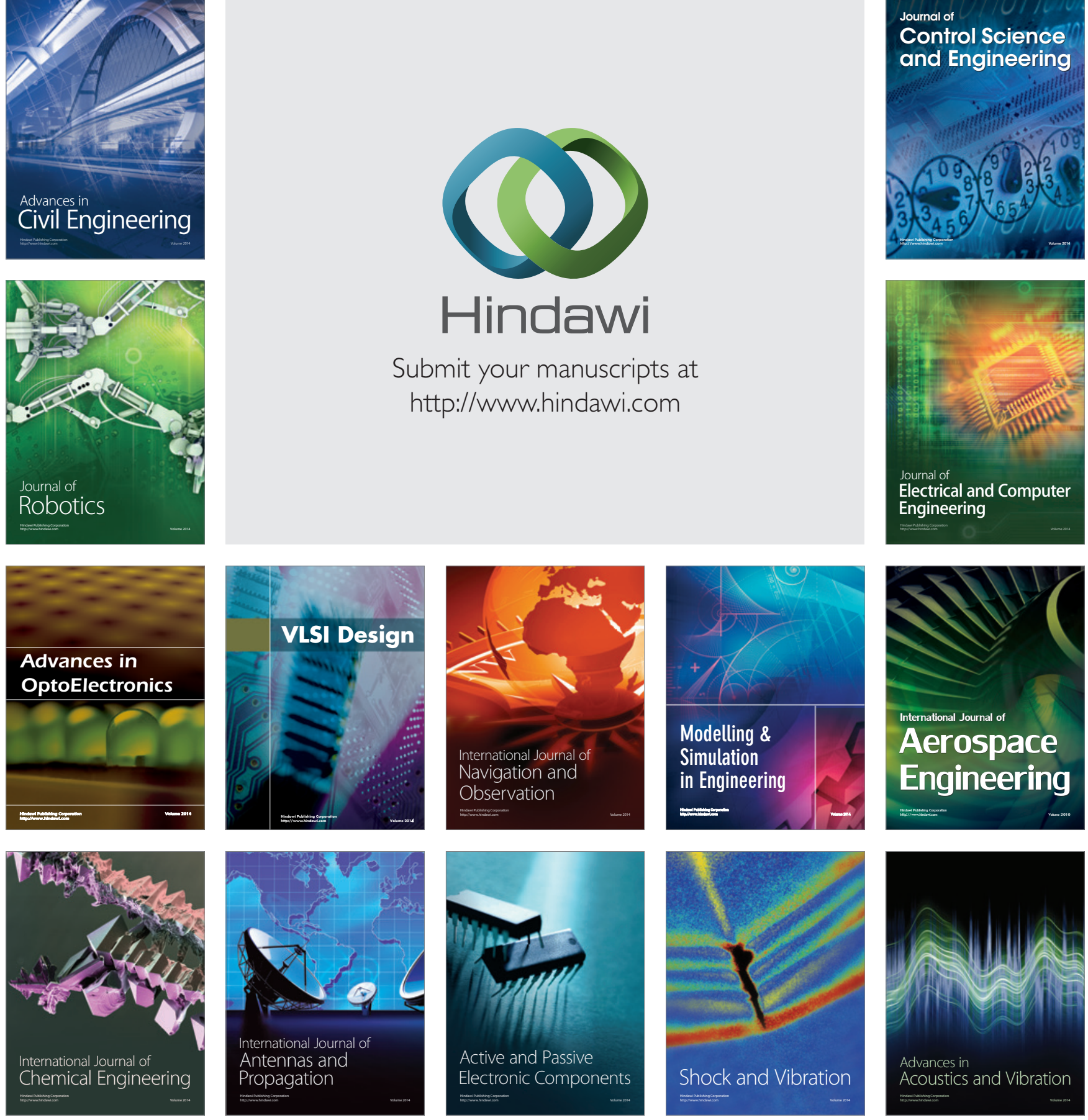